\title{
microRNA-200c downregulates XIAP expression to suppress proliferation and promote apoptosis of triple-negative breast cancer cells
}

\author{
YI REN ${ }^{1,2^{*}}, \mathrm{XUEDONG} \mathrm{HAN}{ }^{2 *}, \mathrm{KUN} \mathrm{YU}^{3}, \mathrm{SU}^{\prime} \mathrm{AN} \mathrm{SUN}^{4}, \mathrm{LINLIN} \mathrm{ZHEN}^{2}, \mathrm{ZHI} \mathrm{LI}^{2}$ and SHUI WANG ${ }^{1}$ \\ ${ }^{1}$ Department of Breast Surgery, The First Affiliated Hospital of Nanjing Medical University, \\ Nanjing, Jiangsu 210029; Departments of ${ }^{2}$ Breast and Thyroid Surgery, ${ }^{3}$ Cardiology and \\ ${ }^{4}$ Pathology, Huai'an First People's Hospital, Huai'an, Jiangsu 223300, P.R. China
}

Received September 11, 2013; Accepted March 21, 2014

DOI: $10.3892 / \mathrm{mmr} .2014 .2222$

\begin{abstract}
Despite advances in the understanding of breast cancer, patients most commonly have a poor prognosis, particularly those with triple negative breast cancer (TNBC). microRNAs (miRNAs) are endogenous non-coding small RNAs, and their aberrant expression is linked to numerous malignancies. In the present study, the expression levels of miR-200c in patients with TNBC were analyzed and it was identified that miR-200c was downregulated in TNBC samples, compared with that in normal adjacent tissues. miR-200c was overexpressed in the TNBC cell line MDA-MB-231 and its functions were studied in vitro and in vivo. An in vitro study revealed that the overexpression of miR-200c inhibited MDA-MB-231 cell proliferation and resulted in the induction of apoptosis. The in vivo data indicated that the overexpression of miR-200c significantly inhibited tumor growth and increased the rate of apoptosis. Target prediction revealed that the X-linked inhibitor of apoptosis (XIAP) had putative complementary sequences to miR-200c, which was confirmed by a dual luciferase reporter assay. Western blot analysis further demonstrated that the expression of XIAP was markedly reduced and that caspase- 3 was highly activated by the overexpression of miR-200c. These findings suggested that miR-200c may function as a tumor suppressor gene in TNBC, at least partly via directly targeting XIAP, and may therefore act as a potential therapeutic target in the development of novel treatment strategies for TNBC.
\end{abstract}

Correspondence to: Professor Shui Wang, Department of Breast Surgery, The First Affiliated Hospital of Nanjing Medical University, 300 Guangzhou Road, Nanjing 210029, P.R. China

E-mail:wso801@163.com

* Contributed equally

Key words: X-linked inhibitor of apoptosis, apoptosis, triple negative breast cancer, microRNA-200c

\section{Introduction}

Breast cancer is one of the most common types of cancer and the second-leading cause of cancer-associated mortality in females worldwide (1). Based on the expression of the estrogen receptor (ER), progesterone receptor (PR) and human epidermal receptor 2 (HER2), breast cancer is categorized into several different subtypes. Of these, $\sim 60-70 \%$ of breast cancer cases express the ER and/or PR, and $20-30 \%$ of breast cancer cases have amplified levels of HER2. These tumors are most commonly treated by targeted therapies. However, tumors lacking ER and PR expression and Her-2 overexpression are referred to as triple-negative breast cancer (TNBC) cases. Due to the lack of targeted treatment options, clinical studies have revealed that TNBC is the most aggressive breast cancer type and is associated with poor prognosis, with the deregulation of apoptosis-associated genes participating in cancer progression and relapse (2).

MicroRNAs (miRNA) are short (19-25 nt) non-coding RNAs, which do not code for proteins but rather regulate mRNA expression at a post-transcriptional level. Since their discovery in 1993, >1,500 human miRNAs have been identified (3). These RNAs directly bind to the 3' untranslated region (UTR) of target mRNAs and facilitate the cleavage or degradation of the transcripts $(4,5)$. Initially, miRNAs were classified as functionless stretches within 'junk DNA'; however, they have recently been described as powerful regulators of gene expression that are associated with cellular development, death and proliferation (6), as well as the pathogenesis of numerous diseases (7). Accumulating evidence has revealed that aberrantly expressed miRNAs participate in tumorigenesis in both a temporal and spatial manner (8). Furthermore, it appears they function as either oncogenes or tumor suppressor genes and regulate a vast range of cellular processes, including cell proliferation, differentiation, apoptosis and metastasis (9). Several studies have identified that the dysregulation of numerous miRNAs, including miR-21 (10), miR-34a (11), miR-101 (12), miR-122 (13) and miR-155 (14), modulates tumorigenic processes in breast cancer. Loss of miR-200 family members (miR-200a, -200b, -200c, -141 and -429), particularly miR-200c, is considered to be crucial in tumor 
progression. Its involvement in epithelial-to-mesenchymal transition (EMT) is well-established; however, its functions in apoptosis are poorly defined.

$\mathrm{X}$-linked inhibitor of apoptosis (XIAP) is the most potent member of the IAP gene family in terms of its ability to inhibit caspase-3, -7 and -9 , and to suppress apoptosis. Due to its potent anti-apoptotic nature, XIAP is a target molecule in the development of therapeutic agents for the treatment of numerous cancer types (15). According to bioinformatic predictions, XIAP is a candidate target gene of miR-200c.

In the present study, the potential associations between miR-200c and XIAP were examined, and its findings may be of clinical utility for the development of novel therapeutic approaches for TNBC treatment.

\section{Materials and methods}

Patients and samples. A total of 20 human TNBC tissues and matched normal adjacent tissues were obtained from the Department of Pathology, Huai'an First People's Hospital (Jiangsu, China). All of the patients or their guardians provided written informed consent and Faculty of Medicine's Ethics Committee of Huai'an First People's Hospital approved all aspects of the present study.

Cell culture andtransfection.Human MDA-MB-231 TNBC cells and human MCF10A mammary epithelial cells were obtained from the Cell Culture Center, Chinese Academy of Medical Sciences (Peking, China). MDA-MB-231 cells were maintained in Dulbecco's modified Eagle's Medium (DMEM) supplemented with $10 \%$ fetal bovine serum and $1 \%$ penicillin/streptomycin. MCF10A cells were maintained in DMEM-F12 medium supplemented with $5 \%$ horse serum, hydrocortisone $(0.5 \mu \mathrm{g} / \mathrm{ml})$, insulin $(10 \mu \mathrm{g} / \mathrm{ml})$, epidermal growth factor $(20 \mathrm{ng} / \mathrm{ml})$ and penicillin-streptomycin $(100 \mu \mathrm{g} / \mathrm{ml})$. Transfection of the miR-200c mimics (Invitrogen Life Technologies, Carlsbad, CA, USA) and the random sequence as a control was performed with Lipofectamine ${ }^{\mathrm{TM}} 2000$ (Invitrogen Life Technologies) according to the manufacturer's instructions and labeled with miR-200c and miR-Ctrl, respectively. Analyses were performed 24-72 $\mathrm{h}$ following transfection.

RNA extraction and quantitative polymerase chain reaction $(q P C R)$. The total RNA from the cell lines and tissues was extracted using TRIzol ${ }^{\circledR}$ (Invitrogen Life Technologies) according to the manufacturer's instructions. miR-200c and RNU6 specific cDNAs were synthesized with the miScript Reverse Transcription kit (Qiagen, Hilden, Germany) according to the manufacturer's instructions. qPCR was performed with the following PCR primers (Invitrogen Life Technologies): miR-200c forward, 5'-CCCTCGTCTTACCCA-3' and reverse, 5'-CCTCCATCATTACCC-3'; RNU6 forward, 5'-GCTTCGGCAGCACATATACTAAAAT-3' and reverse, 5'-CGCTTCACGAATTTGCGTGTCAT-3'. The miScript SYBR-Green PCR kit (Qiagen) was employed to detect the expression levels of these genes. The reaction was conducted according to the manufacturer's instructions. The expression analysis was performed in triplicate for each sample and the relative expression levels compared with RNU6 were calculated using the comparative $\mathrm{Ct}$ method.
Colony formation assay. For the colony formation assay, $1 \times 10^{3}$ miR-200c- or miR-Ctrl-transfected cells were seeded into six-well plates and maintained in media containing $10 \% \mathrm{FBS}, 100 \mathrm{U} / \mathrm{ml}$ penicillium and $100 \mu \mathrm{g} / \mathrm{ml}$ streptomycin for two weeks. The colonies were stained with $0.1 \%$ crystal violet (Sigma, St. Louis, MO, USA) in phosphate-buffered saline (PBS) for $1 \mathrm{~h}$. Triplicate wells were measured in each group. Colony formation was determined by counting the number of stained colonies.

Cell proliferation assay. To evaluate cell proliferation, $3 \times 10^{3}$ MDA-MB-231 cells/well were plated into 96-well plates and transfected with miR-200c mimics or negative control. At 24, 48, 72 and $96 \mathrm{~h}$ following transfection, cell proliferation was measured using the MTT assay kit (Merck Millipore, Billerica, MA, USA) according to the manufacturer's instructions. The optical density (OD) value at $570 \mathrm{~nm}$ was measured using a Synergy HT microplate reader (Bio-Tek Instruments, Inc., Winooski, VT, USA). The experiment was repeated three times.

Fluorescence-activated cell sorting (FACS) assay. A total of $48 \mathrm{~h}$ following transfection, miR-200c or miR-Ctrl cells were washed two times with PBS and incubated with Annexin V-fluorescein isothiocyanate (FITC) and propidium iodide (PI) (BD Pharmingen, San Diego, CA, USA) staining solutions in the dark for $15 \mathrm{~min}$ at $4^{\circ} \mathrm{C}$, then the cells were analyzed using a BD Accuri ${ }^{\mathrm{TM}}$ C6 cytometer (BD Biosciences, Franklin Lakes, NJ, USA).

Western blot analysis. The cultured cells were lysed using radioimmunoprecipitation lysis buffer with a protease inhibitor cocktail tablet ( $2 \mathrm{mM}$ AEBSF, $0.3 \mu \mathrm{M}$ aprotinin, $130 \mu \mathrm{M}$ bestatin, $1 \mathrm{mM}$ EDTA, $14 \mu \mathrm{M}$ E-64, $1 \mu \mathrm{M}$ leupeptin; Sigma, St. Louis, MO, USA). GAPDH was used as an endogenous normalizer. Cell lysates, containing equal amounts of protein, were mixed with SDS protein gel loading solution, resolved by SDS-PAGE and transferred to nitrocellulose membranes. The membranes were blocked in $5 \%$ bovine serum albumin (BSA), and then anti-GAPDH antibody (polyclonal rabbit antiGAPDH), anti-XIAP antibody (polyclonal rabbit anti-XIAP) and anti-Caspase 3 (monoclonal rabbit anti-Caspase 3) antibodies (1:200; Cell Signaling Technology, Inc., Danvers, MA, USA) were added, followed by overnight incubation at $4^{\circ} \mathrm{C}$. The corresponding IRDye 800-labeled secondary antibodies $(1: 10,000)$ were added, followed by overnight incubation at $4^{\circ} \mathrm{C}$. Following washing with Tris-buffered saline with Tween 20, the membrane was scanned using the Odyssey Infrared Imaging System (Li-Cor Biosciences, Lincoln, NE, USA).

Luciferase assay. The 3'UTR of XIAP was amplified from the MDA-MB-231 cell cDNA by PCR. Amplified products were cloned into the corresponding site of the pMIR-REPORT vector (Invitrogen Life Technologies). The mutated 3'UTR of XIAP was synthesized (Invitrogen Life Technologies). The amplified products were cloned into the corresponding site of the pMIR-REPORT vector. The reporter plasmids with wild-type 3'UTR or mutated 3'UTR were termed pMIR-XIAP-wt or pMIR-XIAP-mut, respectively. For the 
A

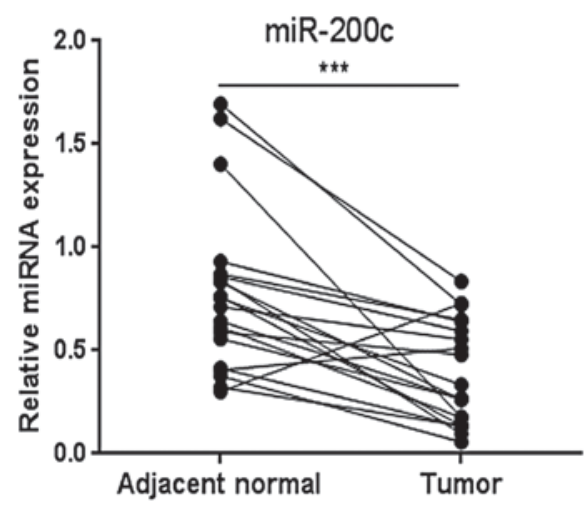

B

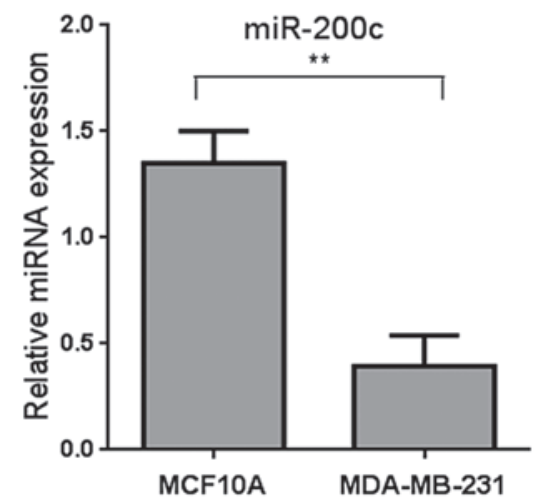

Figure 1. miR-200c expression is downregulated in TNBC tissues and cell lines. (A) Expression of miRNA-200c in 20 paired TNBC tissues and adjacent normal breast tissues. (B) Levels of miRNA-200c in MDA-MB-231 cells and human MCF10A mammary epithelial cell lines. Data are expressed as the mean \pm standard deviation $\left({ }^{* *} \mathrm{P}<0.01 ;{ }^{* * *} \mathrm{P}<0.001\right)$. TNBC, triple negative breast cancer; miRNA, microRNA.

luciferase assay, MDA-MB-231 cells were co-transfected with pMIR-XIAP and the indicated RNAs (miR-200c mimics, negative control) in 24-well plates using Lipofectamine ${ }^{\mathrm{TM}} 2000$. The pMIR-REPORT $\beta$-galactosidase control vector was transfected and served as a control. Firefly and Renilla luciferase activities were measured using a Dual Luciferase Assay system (Promega, Corp., Madison, WI, USA) according to the manufacturer's instructions. The results were expressed as relative luciferase activity (Firefly LUC/Renilla LUC). Each experiment was performed in triplicate for biological repeats.

Mice tumor model. All animal experiments were approved by the Institutional Animal Care and Use Committee of Nanjing Medical University. Following transfection, MDA-MB-231 cells $\left(5 \times 10^{6}\right)$ were suspended with $100 \mu 1$ PBS and injected subcutaneously into the mammary fat pad of female Nod/Scid mice (SLACCAS Laboratory Animal Co., Shanghai, China) at 4-6 weeks of age. The animals were divided into groups of five for each experimental condition. Tumor growth was observed for four weeks following injection. The animals were then sacrificed and the tumors were dissected thoroughly. The tumor volume was calculated using the formula: $\mathrm{V}=$ length $\mathrm{x}$ width ${ }^{2} / 2$.

Terminal deoxynucleotidyltransferase-mediated dUTP nick-end labeling (TUNEL) assay. The mice tumor specimens were immersed and fixed for $2 \mathrm{~h}$ in an ice-cold solution of proteolipid protein, followed by dehydration in ice-cold PBS containing $20 \%$ sucrose for $12 \mathrm{~h}$. After the specimens were embedded in OCT (Sakura Finetek, Torrance, CA, USA), $6 \mu \mathrm{m}$ slices were cut using a cryostat for the TUNEL assay. The slices were blocked with $3 \%$ bovine serum albumin for $1 \mathrm{~h}$ and permeabilized with $0.25 \%$ Triton X-100 for 20 min. A TUNEL Alexa Fluor Imaging kit (Invitrogen Life Technolgoies) was used for TUNEL labeling according to the manufacturer's instructions. The slides were mounted in Vectashield mounting medium with DAPI. The apoptotic fluorescent cells were counted under a confocal microscope and the numbers were expressed as the percentage of total cells \pm standard deviation. A negative control without the enzyme treatment and a positive control with DNase I treatment were also assessed.
Statistical analysis. All statistical analyses were performed using SPSS 17.0 (SPSS, Inc., Chicago, IL, USA). All data were expressed as the mean \pm standard deviation of at least three independent experiments. The differences between the groups were analyzed using the Student's t-test. $\mathrm{P}<0.05$ was considered to indicate statistically significant differences.

\section{Results}

miR-200c is downregulated in TNBC tissues and cell line. To investigate the involvement of miR-200c in human TNBC development, miR-200c expression levels in $20 \mathrm{TNBC}$ tissues and matched normal adjacent tissues were examined by qPCR. As shown in Fig. 1A, the expression of miR-200c was significantly reduced in TNBC tissues compared with the adjacent tissues. Consistently, it was identified that miR-200c was also downregulated in the TNBC cell line MDA-MB-231 compared with that in the human MCF10A mammary epithelial cell line (Fig. 1B).

miR-200c suppresses cell proliferation, colony formation and increases apoptosis in vitro. To determine the effects of miR-200c on cell proliferation, colony formation and apoptosis in vitro, qPCR was used to validate the expression of miR-200c in MDA-MB-231 cells following transfection with a miR-200c mimic. The miR-200c expression levels were significantly upregulated (by 20 -fold) in miR-200c cells compared with the miR-Ctrl cells (Fig. 2A). It was hypothesized that miR-200c may be involved in mediating cancer cell proliferation and apoptosis. To examine this hypothesis, MTT, colony formation and FACS assays were performed to examine the effects of miR-200c on MDA-MB-231 cells. The proliferation of miR-200c or miR-Ctrl cells, as determined by the MTT assay, demonstrated that miR-200c evidently reduced the growth of MDA-MB-231 cells 48 and $72 \mathrm{~h}$ following transfection $(\mathrm{P}<0.05$; Fig. $2 \mathrm{~B})$. A colony formation assay was further performed to investigate the effect of miR-200c on the growth of MDA-MB-231 cells. As demonstrated in Fig. 2C, the colony size and number of miR-200c cells were significantly lower than those of miR-Ctrl cells $(\mathrm{P}<0.01)$. The effect of miR-200c on apoptosis 
A

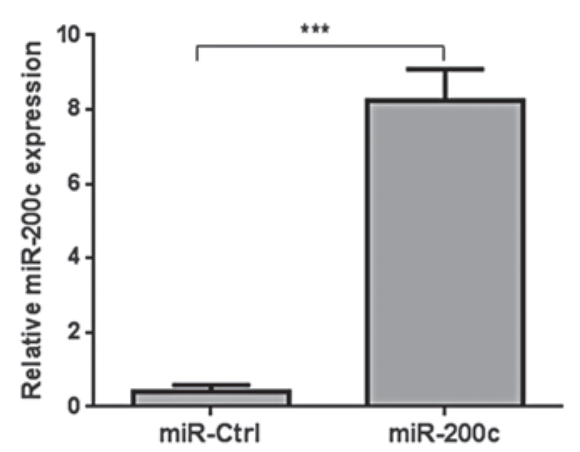

C

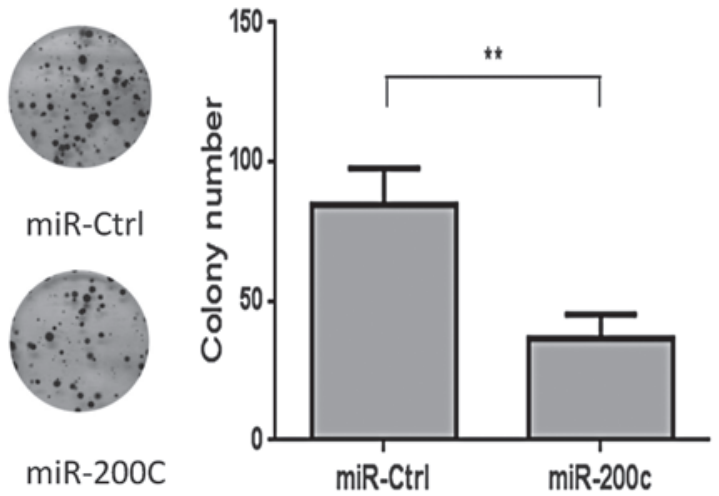

B

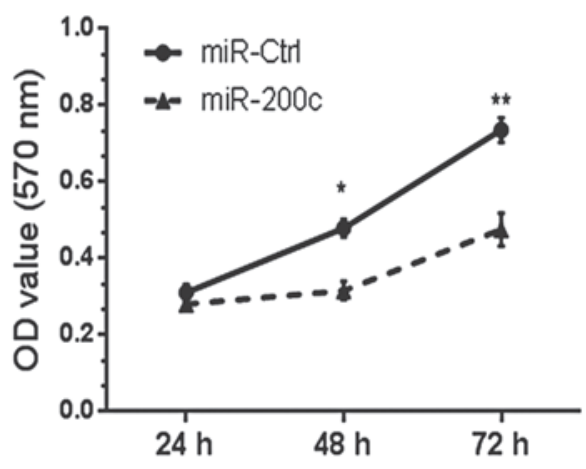

D

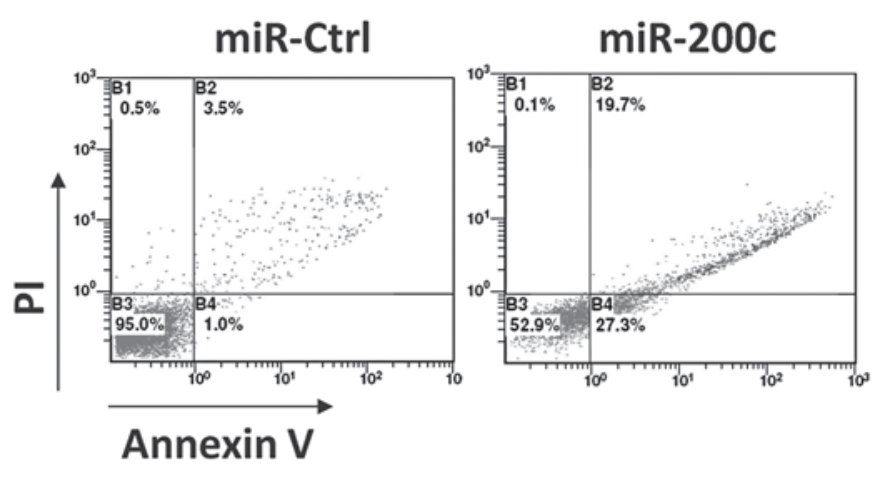

Figure 2. miR-200c suppresses cell proliferation and colony formation, and increases apoptosis in vitro. (A) Relative expression levels of miR-200c in MDA-MB-231 cells transfected with miR-200c mimics or the negative control miR-Ctrl were determined by quantitative polymerase chain reaction. (B) MTT assays were performed to examine the proliferation of miR-200c- or miR-Ctrl-transfected cells at 24, 48 and $72 \mathrm{~h}$ following transfection. (C) The growth capacity of miR-200c- and miR-Ctrl-transfected cells in vitro were assessed by the colony formation assay. (D) miR-200c and miR-Ctrl-transfected cells were stained with Annexin V/propidium iodide (PI) to examine the ratio of cells in early apoptosis. Data are expressed as the mean \pm standard deviation ("P $<0.05$, $\left.{ }^{* *} \mathrm{P}<0.01,{ }^{* * *} \mathrm{P}<0.001\right)$. TNBC, triple negative breast cancer; miRNA, microRNA; OD, optical density.

of MDA-MB-231 cells was determined by flow cytometry using Annexin V-FITC/PI staining. The apoptosis ratio of miR-200c cells was $20.34 \pm 3.27 \%$, which was significantly higher than in the miR-Ctrl cells (Fig. 2D). Taken together, these results indicated that miR-200c significantly induced apoptosis, decreased the proliferation of MDA-MB-231 cells and may have an important role in suppressing the growth of TNBC.

miR-200c suppresses the growth of TNBC tumor and increase apoptosis in vivo. Following the in vitro investigations, it was examines whether miR-200c overexpression may inhibit tumor growth in vivo. To investigate this, an in vivo breast cancer xenograft model in Nod/Scid mice was used. miR-200c or miR-Ctrl cells were injected subcutaneously into the mammary fat pad of female Nod/Scid mice and the tumor sizes were regularly measured. As demonstrated in Fig. 3A, the tumors generated from miR-200c cells were significantly smaller, compared with those generated from the miR-Ctrl cells. The volume curves demonstrated that overexpression of miR-200c markedly inhibited tumor growth in vivo. Based on the in vitro data, it was hypothesized that apoptosis may have a role in miR-200c-mediated tumor suppression. The TUNEL assay was used to detect the apoptotic nuclei in the tumors and the results demonstrated that there were more TUNEL-positive nuclei in the miR-200c group compared with the miR-Ctrl group (Fig. 3B).

$X I A P$ is a direct target of miR-200c. To examine how miR-200c accelerated the apoptosis of MDA-MB-231 cells apoptosis and inhibited tumor growth, public algorithms for prediction analysis (TargetScan and miRBase) were utilized to analyze the potential targets of miR-200c. It was identified that XIAP is a potential target of miR-200c and that the 3'UTR of the XIAP mRNA contains a miR-200c complementary binding site. It was therefore hypothesized that miR-200c inhibits tumor growth by targeting XIAP in breast cancer cells. To further verify this hypothesis, a dual luciferase reporter assay was performed. As shown in Fig. 4A, there was a significant reduction in luciferase activity following co-transfection with miR-200c mimics, compared with the negative control in the pMIR-XIAP-wt group. By contrast, a decrease in luciferase activity was not observed in the pMIR-XIAP-mut group. These results indicated that miR-200c may specifically bind to the 3'UTR of XIAP. Following this, the correlation between miR-200c and XIAP expression in MDA-MB-231 cells was investigated. Following transfection with the miR-200c mimics, the expression of the XIAP protein was evidently reduced, as compared with the negative control. This further activated the 
A miR-Ctrl miR-200c
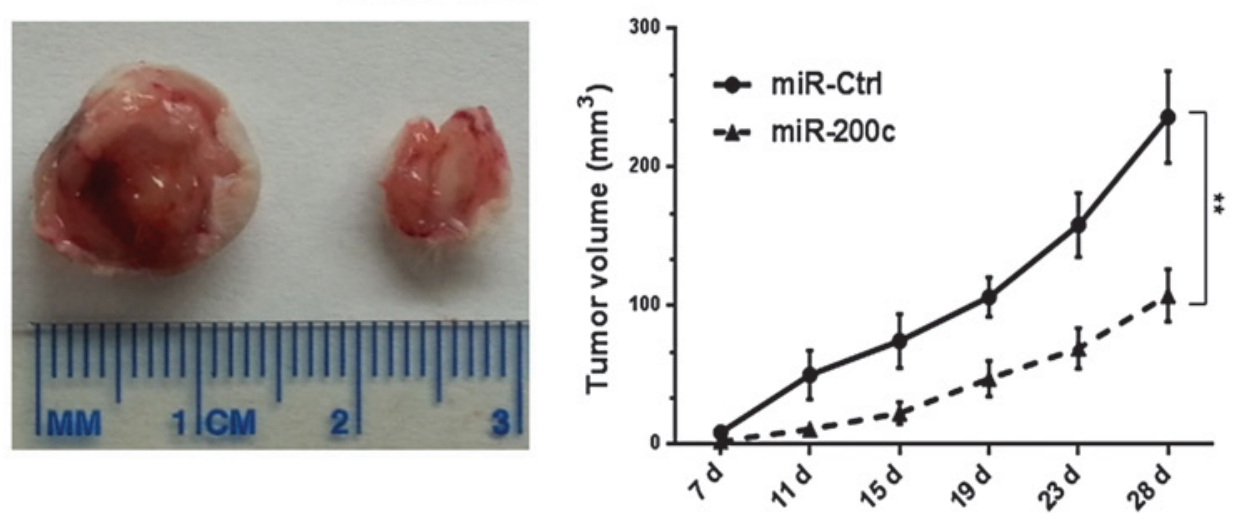

B

DAPI
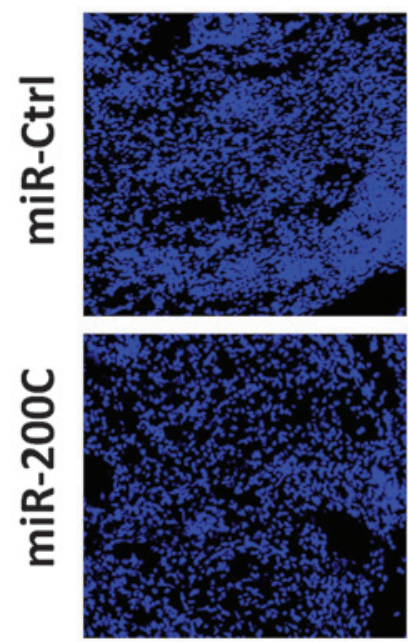

TUNEL
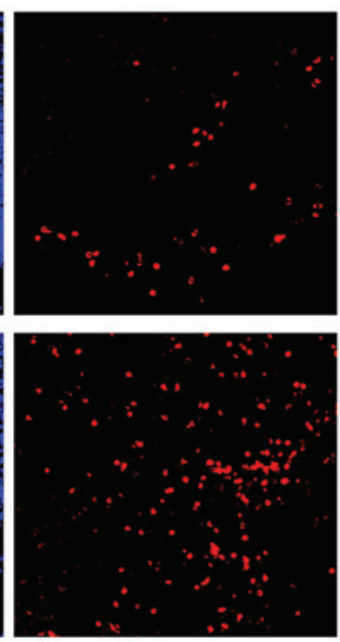

Merge

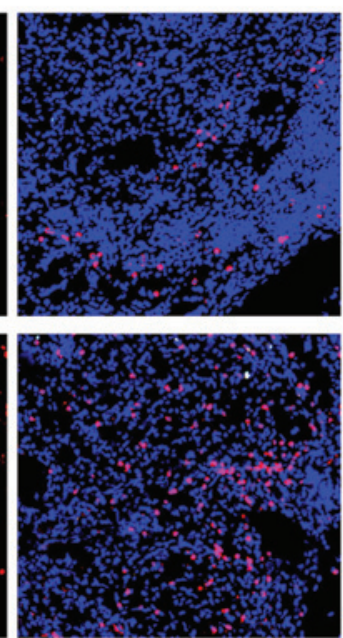

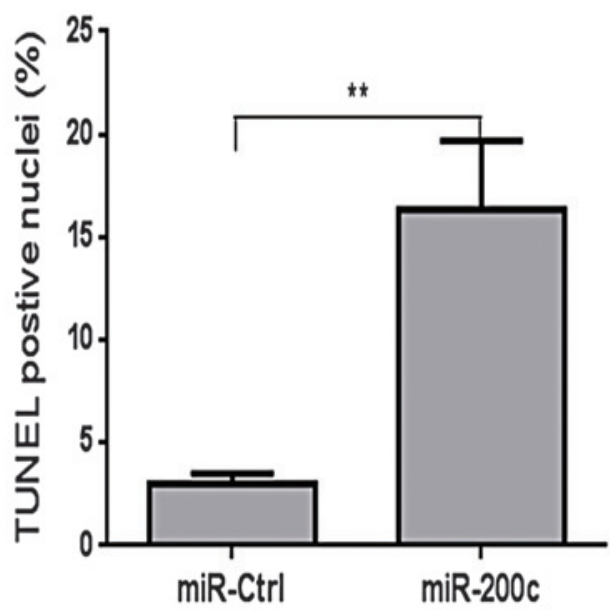

Figure 3. miR-200c suppresses the growth of TNBC tumors and increases apoptosis in vivo. (A) miR-200c- or miR-Ctrl-transfected cells were subcutaneously injected into the mammary fat pad of female Nod/Scid mice. The average tumor volumes were assessed, starting from the tumors formed and continuing until four weeks following injection. (B) TUNEL assay was used to detect the apoptotic nuclei in xenograft tumor tissues. Red fluorescein staining indicates apoptotic cells. DAPI staining was used to determine the number of nuclei and to assess the gross cellular morphology. Data are expressed as the mean \pm standard deviation $\left({ }^{* *} \mathrm{P}<0.01\right)$. TNBC, triple negative breast cancer; miRNA, microRNA; TUNEL, terminal deoxynucleotidyltransferase-mediated dUTP nick-end labeling.

apoptosis pathway and the expression of cleaved caspase-3 protein was significantly increased (Fig. 4B).

\section{Discussion}

Since the discovery of the first miRNA in caenorhabditis elegans, miRNAs and their regulatory functions have been further elucidated, and a fundamental role for miRNA-guided post-transcriptional regulation of gene expression has been revealed (16). Emerging data indicate that dysregulation of miRNAs is associated with almost every type of cancer, including lung (17), papillary thyroid carcinoma (18), colon (19) and breast cancer (20). Iorio et al (21) revealed that miR-10b, miR-125b and miR-145 were downregulated, whereas miR-21 and miR-155 were upregulated in breast cancer tissues, suggesting that they may potentially act as tumor suppressor genes or oncogenes, respectively. Breast cancer comprises three general molecular subtypes based on routine histological/pathological biomarkers, including ER, PR and HER2. Blenkiron et al (22) analyzed the miRNA expression in 93 primary human breast tumors according to the breast cancer subtypes. Of note, a number of miRNAs were considered to be associated with the molecular subtypes of breast cancer and individual miRNAs correlated with clinical pathological factors. Notable effort has focused on the study of the correlation between TNBC and miRNAs to develop targeted therapeutic strategies. Wang et al (23) identified that miR-203 suppressed cell proliferation and migration by targeting survivin and LIM and SH3 domain protein 1 in human TNBC cells. A recent study revealed that the expression of the miRNA-200 family was closely associated with distinct breast cancer phenotypes. Within this family, miR-200c is downregulated in TNBC compared with that in ER/PR positive and HER2 enriched tumors (24). In the present study, 20 TNBC and matched normal adjacent tissues were analyzed by qPCR and it was identified that 
A

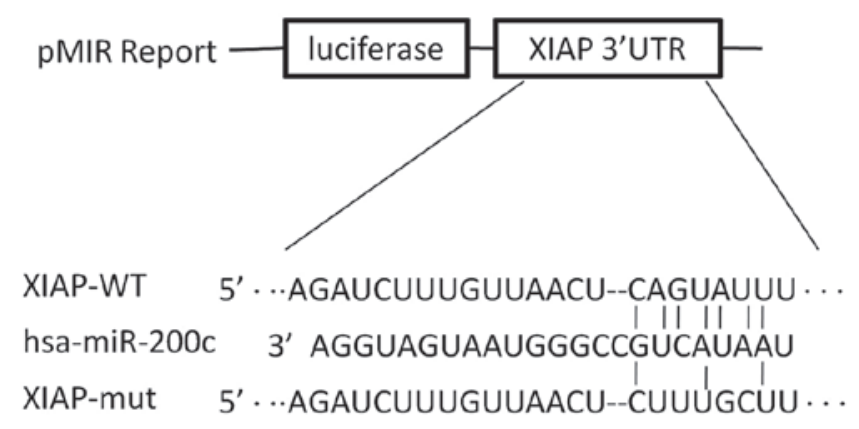

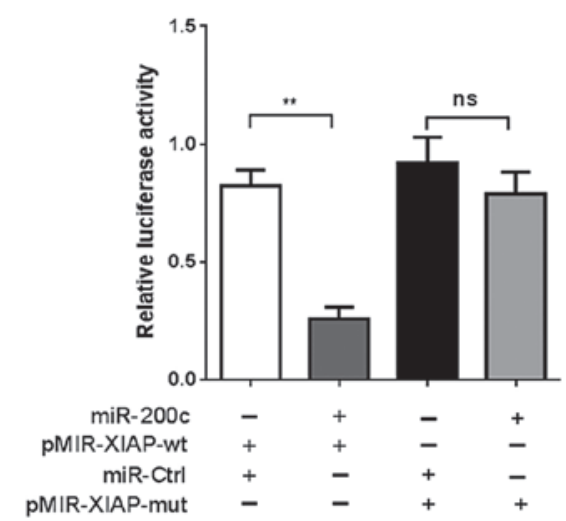

\section{B}

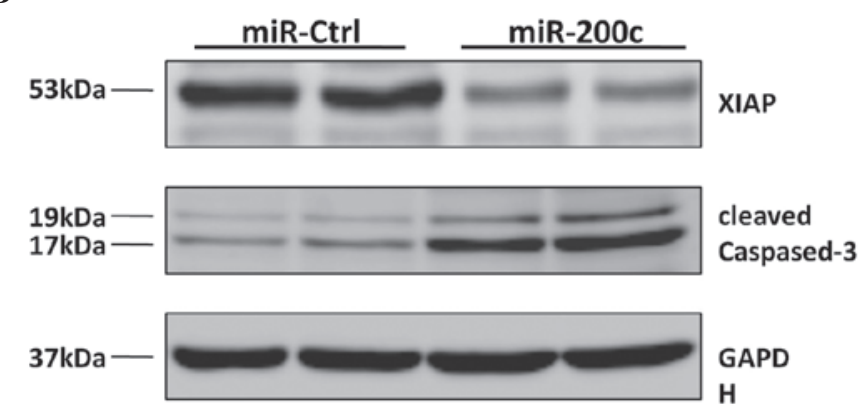

Figure 4. XIAP is a direct target of miR-200c in TNBC cells. (A) A human XIAP 3'UTR fragment containing the WT or mut miR-200c binding sequence was cloned downstream of the luciferase reporter gene. The luciferase reporter plasmid containing WT or mut XIAP 3'UTR was co-transfected into MDA-MB-231 cells with the miR-200c mimics or the negative control. Luciferase activity was determined by the dual luciferase assay and demonstrated as the relative firefly activity normalised to Renilla activity. (B) The expression of XIAP and caspase-3 protein was analyzed by western blot analysis. GAPDH was used as a control. Data are expressed as the mean \pm standard deviation $\left({ }^{* *} \mathrm{P}<0.01\right)$. XIAP, X-linked inhibitor of apoptosis; TNBC, triple negative breast cancer; miRNA, microRNA; WT, wild-type; mut, mutant; ns, not significant; 3'UTR, 3' untranslated region.

miR-200c expression was significantly downregulated in the TNBC samples $(\mathrm{P}<0.05)$.

The miR-200 family members are important tumor-suppressive miRNAs. Aberrant expression of the miR-200 family has been associated with different types of cancers in multiple studies, however, often with contradictory findings. In particular, inconsistencies have emerged in defining their effectors of the EMT occurring in the context of carcinogenesis and embryonic development. Gregory et al (25) reported that miR-200 and miR-205 may inhibit the zinc finger E-box-binding homeobox 1 (ZEB1) and ZEB2, and thus maintain the epithelial cell phenotype. It has been demonstrated that miR-200c is the only miRNA that regulates E-cadherin transcriptional repressor ZEB1 and restores E-cadherin expression in breast cancer cells (26). In addition, ectopic overexpression of miR-200c in embryonal carcinoma cells resulted in neural differentiation and suppressed tumorigenicity of breast CSCs in vivo (27). These studies suggest that miR-200c is an important tumor suppressor in breast cancer, that is significant in the carcinogenesis and the metastatic cascades.

In the present study, a highly aggressive human TNBC cell line, MDA-MB-231, was utilized to examine the regulatory role of miR-200c in TNBC cells. It was demonstrated that the overexpression of miR-200c suppressed cell proliferation and colony formation and induced apoptosis. Then, MDA-MB-231 cells were subcutaneously injected into Nod/Scid mice to resemble a model of human TNBC. The results demonstrated that the miR-200c overexpressing group had a reduced tumor growth rate as compared with the miR-Ctrl in vivo. Consistent with the in vitro results, the number of apoptotic cells was markedly increased in the miR-200c group, as demonstrated by a TUNEL assay. Therefore, the present study provided evidence that miR-200c possessed tumor suppressor activity in TNBC and suggested that the repression of miR-200c may promote tumorigenesis, at least partially by inhibiting breast cancer cell apoptosis. At present, the lack of knowledge regarding specific miRNA gene targets hampers completely understanding the biological functions deregulated by miRNA aberrant expression. To partially overcome this limitation, computational approaches were used to predict gene targets. Various cancer-associated genes are potentially regulated by miR-200c aberrantly expressed in TNBC. In particular, XIAP was of interest, as it is an important apoptosis inhibitory protein and is consistently upregulated in breast cancer.

As it is well-established, apoptosis is an intrinsic cell death mechanism in mammalian cells, but is often defective in cancer cells. The inhibitors of apoptosis (IAP) proteins are characterized by the presence of at least one baculovirus IAP repeat structural domain. Among the IAP members, XIAP is a critical barrier to apoptosis induction in cancer cells due to its robust ability to bind and inhibit initiator caspase- 9 , and effector caspases 3 and 7 (28). Therefore, it effectively functions to 
prevent cell death activation and sustain the survival of cancer cells. XIAP has been demonstrated to be highly expressed in several malignancies, including breast (29), pancreatic (30) and cervical cancer types (31), and has an important role in regulating both apoptosis and cell proliferation. Recently, several studies have revealed that certain miRNAs may directly bind to the 3'UTR of XIAP to inhibit the expression of XIAP and promote apoptosis, including miR-7 (32), miR-24 (33) and miR-513a-5p (34). It is therefore warranted to consider that miR-200c possesses tumor suppressor activity in TNBC at least partially by affecting XIAP expression. In the present study, this hypothesis was confirmed by dual-luciferase activity assays. The XIAP protein expression was downregulated in miR-200c cells compared with that in miR-Ctrl cells, as verified by western blot analysis, and as a result, its downstream pro-apoptotic protein caspase-3 was indirectly activated by miR-200c.

In conclusion, the present study demonstrated that miR-200c was downregulated in TNBC specimens compared with normal adjacent tissues. miR-200c directly reduced the expression of XIAP and then activated the caspase-3-dependent apoptosis pathway, inhibiting TNBC cell proliferation and inducing TNBC cell apoptosis. These data indicated that miR-200c served as a tumor suppressor gene involved in TNBC pathogenesis. The present study also provided a strategy for targeting the miR-200c/XIAP interaction as a novel therapeutic application to treat TNBC.

\section{Acknowledgements}

The present study was supported by the Science and Technology Support Program of Huai'an City of Jiangsu Province (no. HASZ2012013) and the Technology Support Foundation of Nanjing Medical University (no. 2012NJMU149).

\section{References}

1. Berry DA, Cronin KA, Plevritis SK, et al; Cancer Intervention and Surveillance Modeling Network (CISNET) Collaborators: Effect of screening and adjuvant therapy on mortality from breast cancer. N Engl J Med 353: 1784-1792, 2005.

2. Toft DJ and Cryns VL: Minireview: Basal-like breast cancer: from molecular profiles to targeted therapies. Mol Endocrinol 25 199-211, 2011.

3. Lee RC, Feinbaum RL and Ambros V: The C. elegans heterochronic gene lin-4 encodes small RNAs with antisense complementarity to lin-14. Cell 75: 843-854, 1993.

4. Bartel DP: MicroRNAs: target recognition and regulatory functions. Cell 136: 215-233, 2009.

5. Kwak PB, Iwasaki S and Tomari Y: The microRNA pathway and cancer. Cancer Sci 101: 2309-2315, 2010.

6. Ambros V: The functions of animal microRNAs. Nature 431: 350-355, 2004.

7. Alvarez-Garcia I and Miska EA: MicroRNA functions in animal development and human disease. Development 132: 4653-4662, 2005.

8. Katada T, Ishiguro H, Kuwabara Y, et al: microRNA expression profile in undifferentiated gastric cancer. Int J Oncol 34: 537-542, 2009.

9. Croce CM: Causes and consequences of microRNA dysregulation in cancer. Nat Rev Genet 10: 704-714, 2009.

10. Yan LX, Wu QN, Zhang Y, et al: Knockdown of miR-21 in human breast cancer cell lines inhibits proliferation, in vitro migration and in vivo tumor growth. Breast Cancer Res 13: R2, 2011.
11. Li L, Xie X, Luo J, et al: Targeted expression of miR-34a using the T-VISA system suppresses breast cancer cell growth and invasion. Mol Ther 20: 2326-2334, 2012.

12. Wang R, Wang HB, Hao CJ, et al: miR-101 is involved in human breast carcinogenesis by targeting Stathmin1. PLoS One 7: e46173, 2012.

13. Wang B, Wang $\mathrm{H}$ and Yang Z: miR-122 inhibits cell proliferation and tumorigenesis of breast cancer by targeting IGF1R. PLoS One 7: e47053, 2012

14. Sun Y, Wang M,Lin G, et al: Serum microRNA-155 as a potential biomarker to track disease in breast cancer. PLoS One 7: e47003, 2012.

15. Danson S, Dean E, Dive C and Ranson M: IAPs as a target for anticancer therapy. Curr Cancer Drug Targets 7: 785-794, 2007.

16. Stefani G and Slack FJ: Small non-coding RNAs in animal development. Nat Rev Mol Cell Bio 9: 219-230, 2008.

17. Yanaihara N, Caplen N, Bowman E, et al: Unique microRNA molecular profiles in lung cancer diagnosis and prognosis. Cancer Cell 9: 189-198, 2006.

18. He H, Jazdzewski K, Li W, et al: The role of microRNA genes in papillary thyroid carcinoma. Proc Natl Acad Sci USA 102: 19075-19080, 2005.

19. Cummins JM, He Y, Leary RJ, et al: The colorectal microRNAome. Proc Natl Acad Sci USA 103: 3687-3692, 2006.

20. Mattie MD, Benz CC, Bowers J, Sensinger K, et al: Optimized high-throughput microRNA expression profiling provides novel biomarker assessment of clinical prostate and breast cancer biopsies. Mol Cancer 5: 24, 2006.

21. Iorio MV, Ferracin M, Liu CG, et al: MicroRNA gene expression deregulation in human breast cancer. Cancer Res 65: 7065-7070, 2005.

22. Blenkiron C, Goldstein LD, Thorne NP, et al: MicroRNA expression profiling of human breast cancer identifies new markers of tumor subtype. Genome Biol 8: R214, 2007.

23. Wang C, Zheng X, Shen C and Shi Y: MicroRNA-203 suppresses cell proliferation and migration by targeting BIRC5 and LASP1 in human triple-negative breast cancer cells. J Exp Clin Cancer Res 31: 58, 2012.

24. Castilla MÁ, Díaz-Martín J, Sarrió D, et al: MicroRNA-200 family modulation in distinct breast cancer phenotypes. PLoS One 7: e47709, 2012.

25. Gregory PA, Bert AG, Paterson EL, et al: The miR-200 family and miR-205 regulate epithelial to mesenchymal transition by targeting ZEB1 and SIP1. Nat Cell Biol 10: 593-601, 2008.

26. Hurteau GJ, Carlson JA, Roos E and Brock GJ: Stable expression of miR-200c alone is sufficient to regulate TCF8 (ZEB1) and restore E-cadherin expression. Cell Cycle 8: 2064-2069, 2009.

27. Wellner U, Schubert J, Burk UC, et al: The EMT-activator ZEB1 promotes tumorigenicity by repressing stemness-inhibiting microRNAs. Nat Cell Biol 11: 1487-1495, 2009.

28. Sun C, Cai M, Gunasekera AH, et al: NMR structure and mutagenesis of the inhibitor-of-apoptosis protein XIAP. Nature 401: 818-822, 1999.

29. Lima RT, Martins LM, Guimarães JE, Sambade C and Vasconcelos MH: Specific downregulation of bcl-2 and xIAP by RNAi enhances the effects of chemotherapeutic agents in MCF-7 human breast cancer cells. Cancer Gene Ther 11: 309-316, 2004.

30. Jiang C, Yi XP, Shen H and Li YX: Targeting X-linked inhibitor of apoptosis protein inhibits pancreatic cancer cell growth through p-Akt depletion. World J Gastroenterol 18: 2956-2965, 2012.

31. Cho EA, Oh JM, Kim SY, Kim Y and Juhnn YS: Heterotrimeric stimulatory GTP-binding proteins inhibit cisplatin-induced apoptosis by increasing $\mathrm{X}$-linked inhibitor of apoptosis protein expression in cervical cancer cells. Cancer Sci 102: 837-844, 2011.

32. Liu S, Zhang P, Chen Z, Liu M, Li X and Tang H: MicroRNA-7 downregulates XIAP expression to suppress cell growth and promote apoptosis in cervical cancer cells. FEBS Lett 587: 2247-2253, 2013.

33. Xie Y, Tobin LA, Camps J, et al: MicroRNA-24 regulates XIAP to reduce the apoptosis threshold in cancer cells. Oncogene 32: 2442-2451, 2013

34. Shin S, Moon KC, Park KU and Ha E: MicroRNA-513a-5p mediates TNF- $\alpha$ and LPS induced apoptosis via downregulation of X-linked inhibitor of apoptotic protein in endothelial cells. Biochimie 94: 1431-1436, 2012. 